\title{
Protecting welfare or obstructing research?
}

Is it or is it not distress? That was the question facing Dr. Sandy Ayers as she was reviewing an IACUC protocol from Great Eastern University. Dr. John Summers, the writer of the protocol, noted that the Guide for the Care and Use of Laboratory Animals (the Guide) ${ }^{1}$ recommended housing mice at a room temperature that was below the lower critical temperature of the animals' thermoneutral zone. Summers neglected to add that the Guide also recommended having adequate resources for thermoregulation available to help protect the mice from cold stress. But perhaps that was not pertinent because cold exposure was the key experimental condition. Further, Summers indicated that his research method gradually adapted healthy young adult animals to progressively lower environmental temperatures, eventually to $7^{\circ} \mathrm{C}$. He stated that he was not aware of any evidence to indicate that adapting mice to low environmental temperatures led to a stress reaction in the animals. In fact, he offered the IACUC data from his own research showing that fecal corticosterone levels from his coldadapted mice were not significantly different during cold exposure versus normal room temperature conditions.

As Ayers reviewed the protocol, she was impressed with the data presented and was about to approve it as written, when she began to have second thoughts. She felt she needed information on how mice that did not adapt to the cold temperature would be identified and removed from the study. She also suggested that the mice should have their body temperature measured on a weekly basis and that any mouse with a body temperature of less than $31^{\circ} \mathrm{C}$ should be removed from the study, as such a finding likely would be indicative of distress.

Do you think Ayers was doing her best to protect the welfare of Summers' animals or do you think Ayers was being so overly cautious that she became an obstruction to research?

1. Institute for Laboratory Animal Research. Guide for the Care and Use of Laboratory Animals (National Academies Press, Washington, DC, 2011).

\section{RESPONSE}

\section{Kudos to Ayers}

\section{Marcy Brown, BS, MA, CPIA \& \\ Nina Hahn, DVM, PhD, DACLAM}

In the scenario described above, the first point to consider is whether the animals will experience distress. US Government Principle IV (ref. 1) states, "unless the contrary is established, investigators should consider that procedures that cause pain or distress in human beings may cause pain or distress in other animals," which suggests that housing mice at temperatures as low as $7{ }^{\circ} \mathrm{C}$ could very well involve distress to the animals. Given this potential for distress, and the fact that the environmental conditions are outside of the temperature standards described in the Guide for the Care and Use of Laboratory Animals ${ }^{2}$ (the Guide), Ayers was not being overly cautious; she was obligated to address these issues. The Public Health Service Policy on Humane Care and Use of Laboratory Animals ${ }^{3}$ and the Animal Welfare Act regulations ${ }^{4}$ contain almost identical language describing the responsibility of the IACUC to critically evaluate all proposed animal activities to ensure that pain and distress are avoided or minimized (e.g., "Procedures with animals will avoid or minimize discomfort, distress, and pain to the animals, consistent with sound research design." ${ }^{2}$ ). The Guide reiterates this responsibility. Additional questions could be asked during the review process to help elucidate the distress level for these animals. For instance, providing deep bedding and housing mice with cage-mates to allow for behavioral thermoregulation would presumably be less stressful than single-housing without nesting material.

Ayers also deserves kudos for recognizing the need for the principal investigator (PI) to identify and monitor humane endpoints. According to the Guide, a description of the criteria and process for timely intervention and removal of animals from the study if pain or distress is anticipated should be included in the protocol by the PI and reviewed by the IACUC. Asking Summers to provide information on how mice that did not adapt to the cold would be identified and removed from the study is therefore appropriate. The Guide also notes that the PI "should identify, explain, and include in the animal use protocol a study endpoint that is both humane and scientifically sound" and that the determination of a humane endpoint should involve the PI, the veterinarian and the IACUC. Here again, Ayers' suggestion that body temperatures be measured weekly and any animals with a body temperature less than $31{ }^{\circ} \mathrm{C}$ be removed from the study is appropriate, at least as a starting point for a discussion to identify humane endpoints that could be described in the protocol.

When laboratory animals are subjected to conditions that cause pain or distress, the benefits of this exposure must outweigh the costs, as a matter of ethics. In this case, there is a clear potential for pain or distress in the animals. It is the responsibility of the investigator to include in the animal use protocol a scientific justification for the need for the procedures (“...their relevance to human or animal health, the advancement of knowledge, or the good of society" ${ }^{1}$ ); to state that he or she has considered all possibilities for minimizing pain and distress that don't 